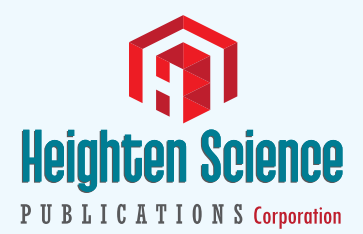

ISSN

2573-7724
*Address for Correspondence: Viviana

Frantellizzi, Viale Regina Elena 324, 00161, Rome Italy, Tel: 0649978573; Fax: 0649978592; Email: viviana.frantellizzi@uniroma1.it

Submitted: 19 July 2018

Approved: 27 July 2018

Published: 28 July 2018

Copyright: @2 2018 De Vincentis G, et al. This is an open access article distributed under the Creative Commons Attribution License, which permits unrestricted use, distribution, and reproduction in any medium, provided the original work is properly cited.

Keywords: Nuclear Medicine; Incidental findings; Risk conditions
Case Report

\section{Incidental findings in traditional nuclear medicine practice}

\author{
Giuseppe De Vincentis ${ }^{1}$, Mariano Pontico ${ }^{1}$, Maria Ricci ${ }^{1}$, \\ Viviana Frantellizzi ${ }^{1,2 *}$ and Mauro Liberatore ${ }^{1}$ \\ 'Department of Radiological Sciences, Oncology and Anatomical Pathology, Sapienza \\ University of Rome, Viale Regina Elena 324, 00161, Rome, Italy \\ ${ }^{2} \mathrm{PhD}$ Program: Angio-Cardio-Thoracic Pathophisiology and Imaging, Sapienza University of \\ Rome, Rome, Italy
}

\section{Abstract}

The presence of an incidental finding, defined as an abnormality which is unrelated to the initial scanning indication, is widely increases due to the access to new devices and imaging modalities. This growing number of incidental findings can lead to additional medical care including unnecessary tests nevertheless, in a minority of patients, can lead to diagnosis of an important and unexpected condition that could be crucial for the patient. We reported three cases in which nuclear medicine imaging, performed for different reasons and showed a relevant and unexpected pathology. In the case 1, a bone scan, performed in a 66 aged woman for breast cancer staging, allowed the diagnosis of a uterine fibroma. In the case 2, a HMPAO labeled-WBC scintigraphy performed because of a suspect of osteomyelitis, showed a remarkable heart-shaped photopenic area, highly suggestive of cardiac global dilatation. In the case 3 , a 62 aged man referred to bone scintigraphy for the staging of recent diagnosed lung cancer. The bone scan allowed the diagnosis of a meningioma. Therefore, the occurrence of incidental findings could lead to reveal relevant abnormalities for the diagnostic pathway.

\section{Introduction}

Imaging techniques play a major role in the management of many patients. The quality of imaging examinations has improved considerably and access to these clinical tools has increased. However, these techniques often give rise to findings that are incidental and unrelated to the reason the study was prescribed. The growing number of imaging techniques performed per patient causes an increase in the number of incidental findings. The description of an unexpected finding can lead to additional medical care including unnecessary tests, other diagnostic procedures and treatments which in some cases may pose an additional and unnecessary risk to the patient $[1,2]$. The absence of evidence from multicenter controlled trials leads to unawareness of the real prognostic significance for incidental findings and subsequent clinical implications. According to a previous multi-center analysis of low-resolution CT attenuation correction images acquired during single-photon emission CT (SPECT-CT), only four $(0.2 \%)$ of these were potentially detrimental to patient outcome, to mean that there was a high suspicion that the pathology, if any, underlying the incidental radiological finding could impact negatively on patient well-being and that further investigation was required [3]. Nevertheless, in some cases, an incidental finding may involve an important condition that could be crucial for the patient. Further studies are needed in order to estimate the additional costs of their imaging workup incurred from subsequent radiologic follow-up evaluation. According to a previous paper, out of 474 patients with an incidental finding, 25 had clinical implications and those with an incidental finding in thoracic cavity or in head/neck were more likely to have additional evaluations [4]. This three cases report was meant to highlight the significance of careful inspection of scintigraphic images to point out collateral finding, which could lead to uncover subclinical relevant disease and alterations, assuming that early diagnosis of pathologies has mostly a crucial clinical impact on the patient's prognosis. 


\section{Case 1: Uterine fibroid on 99mTc-HDP bone scan}

In this case we report a 66 aged woman with diagnosis of breast cancer came to our center for the staging of the disease. Therefore, a $99 \mathrm{mTc}$-byphosphonates bone scintigraphy have been performed in order to focus on eventual secondary lesions. Concerning the main clinical request, the bone scan whole body image, obtained 2 hours after the i.v. injection of the osteotropic radiopharmaceutical, did not show any pathologic uptake of the tracer in the skeleton. Conversely, focusing on the pelvis region, it was evident an area with very faint fixation able to distort the profile and the shape of the full urinary bladder (Figure 1). Moreover, this incidental finding determined an $a b$ extrinseco compression on the superior wall of the bladder. The detailed spot view, obtained after urination, confirmed the profile alteration of the bladder on the superior right side. The woman had no previous significant issues in gynecologic past and recent anamnesis and, moreover, the absence of reported clinical symptoms related to gynecologic disease didn't induce the suspect of an altered gynecologic condition. This incidental finding required further investigations to assess their clinical relevance, therefore it was suggested a complementary morpho-structural examination by pelvic transvaginal ultrasound. She underwent both ultrasound and further morphostructural exam (MRI) brought out a notable mass in the context of uterine wall that was reported as a uterine fibroid or leiomyoma.

\section{Case 2: Cardiac dilatation on 99mTc-HMPAO labeled-WBC scintigraphy}

A 72 years aged woman, in treatment for arterial hypertension and overweight, was referred to $99 \mathrm{mTc}$-HMPAO labeled-WBC scintigraphy to assess a suspect of knee prosthesic joint infection, supported by recurrent gonalgia and functional impairment together with C-reactive protein (CRP) elevation, even after antibiotic therapy. The arhtroplasty surgery took place about nine months earlier, preceded by standard preoperative screening for cardiovascular risk: the cardiac ultrasound and ECG exam did not show any significant abnormalities, with exception of mild aortic valve sclerosis and, furthermore, the systolic function was normal, and no dilatation was reported. According guidelines [5], a scintigraphic spot of the thorax have been performed for "in vivo" quality controls (Figure 2). In fact, lung images acquired at 30 min after injection should show an almost complete clearance of lung activity, while focal spots or diffuse spots of radioactivity in the lungs at $30 \mathrm{~min}$ or later indicate errors in the radiolabelling process, as clumps of cells in the injection sample or cells damages. The scintigraphic

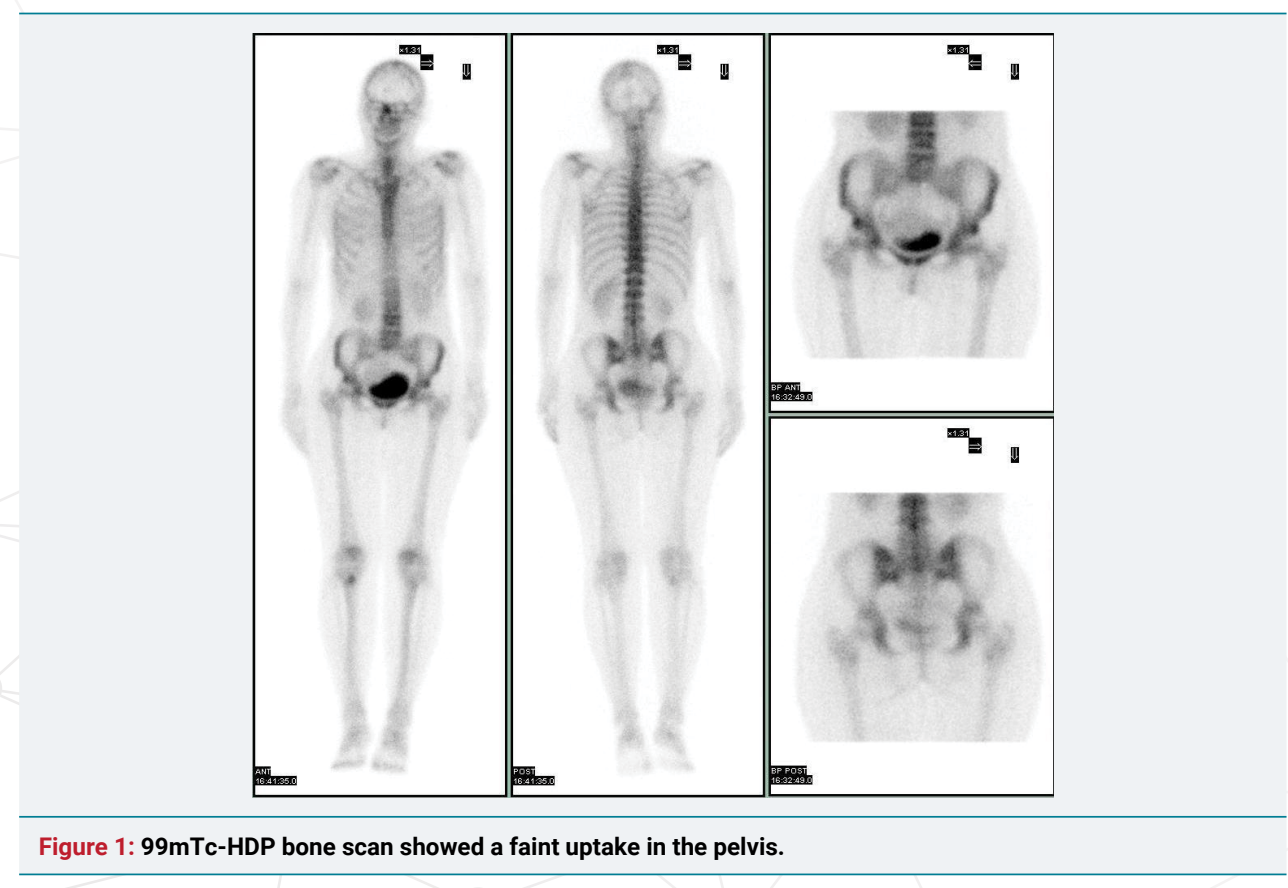




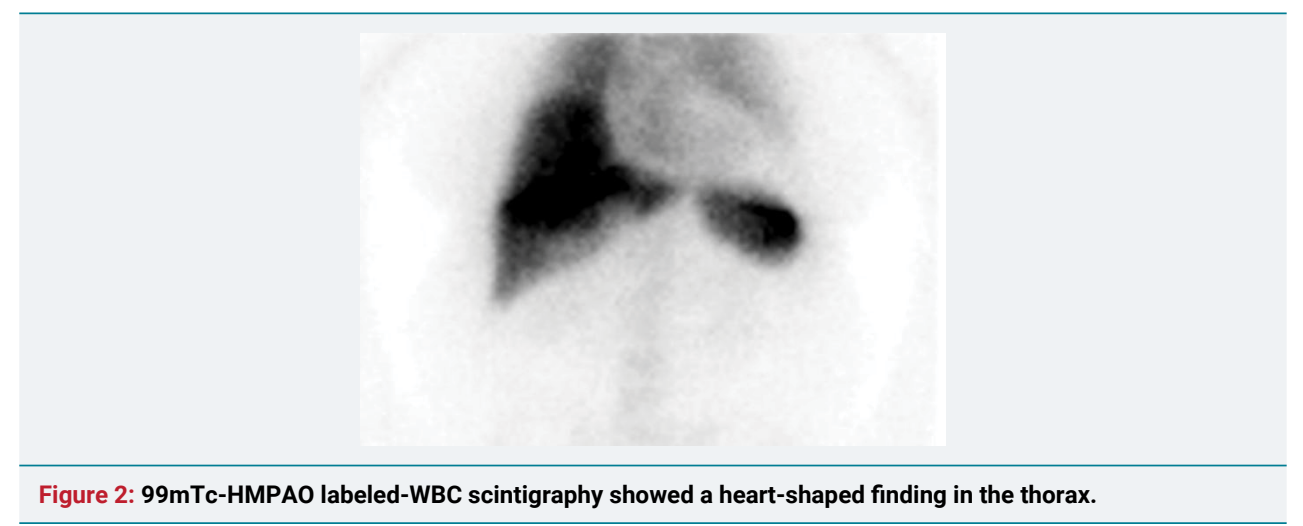

spot of the thorax at $30 \mathrm{~min}$ after injection reported photopenic area in the cardiac region and, therefore, a diagnostic image acquisition at 1 hour after injection has been subsequently performed, in addiction to knee scintigraphic spot. It should be noticed that an additional scintigraphic spot doesn't grow the radiological risk of the patient, because of administered radio-activity is represented by the radiolabelled compound injection, therefore it isn't influenced by further spots image acquisitions. The spot view scintigraphic image of the thorax at 1 hour after injection, showed a remarkable heart-shaped photopenic area in the cardiac region, evidently enlarged compared to that of a healthy one. This incidental finding was highly suggestive of cardiac global dilatation. Therefore we suggested to perform an additional ultrasound exam that reported the presence of a significant pericardial effusion, not present at previous imaging test, deserving further cardiologic investigation.

\section{Case 3: Meningioma on 99mTc-HDP bone scan}

A 62-aged man referred to $99 \mathrm{mTc}$-byphosphonates bone scintigraphy for the staging of recent diagnosed lung cancer. Concerning the main clinical request, the bone scan whole body image, obtained 2 hours after the i.v. injection of the osteotropic radiopharmaceutical, did not show any pathologic uptake of the tracer in the skeleton, excluding an uptake in L4 vertebrae, potentially due to osteodegeneration. Conversely, focusing on the head region, it was evident a focal area of increased uptake in para-median left frontal area (Figure 3). The man had no previous significant neurologic symptoms in recent anamnesis. Both the locus of this uptake (frontal bone or soft tissues) and the cause of this uptake were unclear, therefore we suggested to perform a complementary morpho-structural examination. The CT scan subsequently performed reported a great meningioma localized in left frontal area, with Calcium deposits included (Figure 4).

\section{Discussion}

The quality of imaging examinations has improved considerably and access to these new devices has increased, assuming that "newer is better". However, these techniques often give rise to findings that are incidental to the reason the study was ordered. The presence of an incidental finding, defined as an abnormality which is unrelated to the initial scanning indication, has been largely known as the potential trigger of a cascade effect in the clinical care of patients [1]. At present, many authors reported the incidence, clinical impact and management of radiologic imaging, mainly during computed tomography and magnetic resonance exams, with heterogeneous results. A previous metanalysis found a high percentage of incidental findings in imaging tests, especially in patients with non-specific initial diagnoses. Most patients with abnormalities were clinically followed up, especially those with findings of minor importance. However, only a minority of them were clinically confirmed [1]. Particularly a multi-center analysis of low-resolution CT attenuation correction incidental findings reported that only four $(0.2 \%)$ of these were potentially detrimental to patient outcome, leading often to an unnecessary cascade effect [3]. Attention must be paid by imaging specialist when 


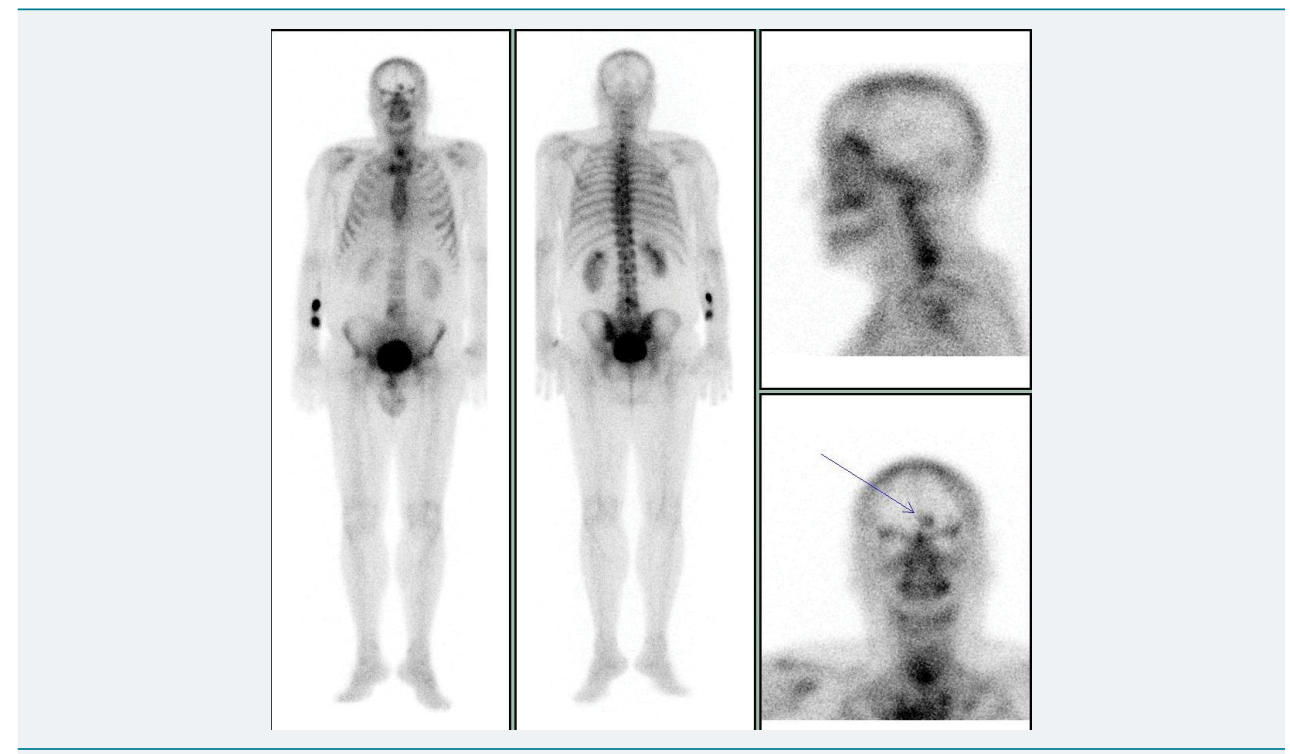

Figure 3: 99mTc-HDP bone scan showed a hot spot in the frontal region.

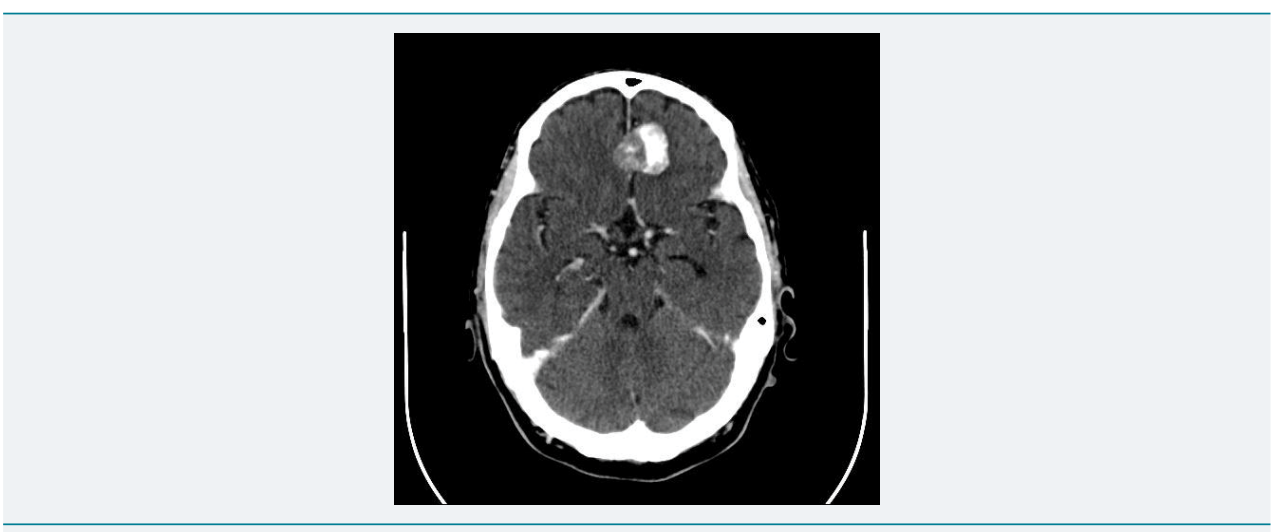

Figure 3: Contrast enhanced CT scan of brain showing the calcified and enhancing meningioma, transversal slice.

Same case of Figure 3.

describing out incidental findings because of they could trigger additional medical care, including even unnecessary tests, diagnostic procedures and treatments which in some cases may consist in an additional risk to the patient. This process has been called the "cascade effect". The potential risk of unexpected findings is an important follow-up, but without obtaining clinical confirmation or clinical impact of these abnormalities. In most recent years, the constantly increasing application of PET-CT imaging in clinical practice expanded this type of reporting even in this nuclear medicine field, in spite of rare cases described in traditional nuclear medicine.

In contrast, in the field of the traditional nuclear medicine imaging there is lack of reporting about the occurrence of incidental findings among the various heterogeneous imaging modalities. The role of the nuclear medicine physician is crucial in deciding whether an image uncommon finding is to consider physiologic or a potentially important diagnostic discovery. Nevertheless, the incidental finding is also a problem for clinicians and the collaboration between nuclear medicine specialists and clinicians is essential to manage the incidental finding issue. Therefore, an ethical framework have been presented in order to guide researchers and research ethics committees in the management of appropriate pathways for the handling of incidental findings in imaging studies. The framework consists of seven steps: anticipation of findings, information provision and informed consent, scan acquisition, review of scans, consultation on detected abnormalities, communication of the finding, and further clinical management and follow-up of the research participant. Each of these steps represents a key decision to be made by researchers, which should be justified not only with reference to costs and/ 
or logistical considerations, but also with reference to researchers' moral obligations and the principle of reciprocity [6]. Nevertheless, in some cases, these incidental findings, especially if confirmed by clinical features, may be crucial in a minority of patients. In fact, in the case 1 reported, the diagnosis of uterine fibroid or leiomyoma had a relevant clinical impact, especially considering the possible complications of this disease, as great hemorrhages risk or implication in fertility. Furthermore, in the case 2 reported, an ultrasound exam allowed the diagnosis of significant pericardial effusion not present at previous imaging test that, if untreated, could potentially lead to severe complications and death. It should be noticed that, in both these cases, a simple and cheap ultrasound exam was able to detect a potentially risk condition. Also in the case 3 reported the incidental finding, due to calcium deposits include in meningioma area, revealed a crucial condition that need a prompt treatment and/or monitoring.

Physicians should be particularly careful in conditions with high rate of relevant findings, as total-body CT scanning in trauma patients [7]. Furthermore, incidental finding in thoracic cavity or in head/neck are more likely to have additional evaluations [4]. Therefore, further papers are necessary in order to identify the most dangerous incidental findings that should be carefully further explored and monitored. Also, the added costs should be considered even if, according a previous paper concerning incidental findings in Low-Dose Spiral CT, just moderate incremental costs are incurred, based on additional radiologic procedures generated during short-term follow-up, given the potential for positive effects on patient care [8].

\section{Conclusion}

The occurrence of incidental findings in nuclear medicine practice could lead to reveal relevant abnormalities for the diagnostic pathway and, even more, for its further clinical significance. Therefore, investigators and institutional review boards must anticipate and articulate plans for handling incidental findings, in order both to avoid cascade effect and to identify potentially risk conditions.

\section{Reference}

1. Lumbreras $B$, Donat $L$, Hernández-Aguado I. Incidental findings in imaging diagnostic tests: a systematic review. Br J Radiol. 2010; 83: 276-289. Ref.: https://tinyurl.com/ya6huxpr

2. Priola AM, Priola SM, Giaj-Levra M, Basso E, Veltri A, et al. Clinical implications and added costs of incidental findings in an early detection study of lung cancer by using low-dose spiral computed tomography. Clin Lung Cancer. 2013; 14: 139-148. Ref.: https://tinyurl.com/y8254vq7

3. Coward J, Lawson R, Kane $T$, Elias $M$, Howes A, et al. Multi-centre analysis of incidental findings on low-resolution CT attenuation correction images. Br J Radiol. 2014; 87: 20130701. Ref.: https://tinyurl.com/ycoe9t4n

4. Lumbreras B, Gonz'alez-Alvarezl, G 'omez-S'aez N, Fermina Lorente M, Hern'andez-Aguado I. Management of patients with incidental findings in imaging tests: a large prospective single-center study. Clin Imaging. 2014; 38: 249-254. Ref.: https://tinyurl.com/ybsqp9tj

5. de Vries EF, Roca M, Jamar F, Israel O, Signore A. Guidelines for the labelling of leucocytes with $(99 \mathrm{~m})$ Tc-HMPAO. Inflammation/Infection Taskgroup of the European Association of Nuclear Medicine. Eur J Nucl Med Mol Imaging. 2010; 37: 842-848. Ref.: https://tinyurl.com/y82gszvc

6. Bunnik EM, van Bodegom L, Pinxten W, de Beaufort ID, Vernooij MW. Ethical framework for the detection, management and communication of incidental findings in imaging studies, building on an interview study of researchers' practices and perspectives. BMC Med Ethics. 2017; 18: 10. Ref.: https://tinyurl.com/yanbhy2j

7. Treskes K, Bos SA, Beenen LFM, Sierink JC, Edwards MJR, et al. High rates of clinically relevant incidental findings by total-body CT scanning in trauma patients; results of the REACT-2 trial. Eur Radiol. 2017; 27: 2451-2462. Ref.: https://tinyurl.com/ycdt7nhe

8. Priola AM, Priola SM, Giaj-Levra M, Basso E, Veltri A, et al. Clinical Implications and Added Costs of Incidental Findings in an Early Detection Study of Lung Cancer by Using Low-Dose Spiral Computed Tomography. Clin Lung Cancer. 2013; 14: 139-148. Ref.: https://tinyurl.com/y8254vq7 